ms

médecine/sciences $1994 ; 10: 107981$

\title{
OBÉSITÉ ET RECHERCHE EN 1994
}

\section{Daniel Ricquier}

\section{ADRESSE}

D. Ricquier : directeur de recherche au Cinrs. Centre de Recherches sur l'Endocrinologie Moléculaire et le Développement, Cnrs, 9, rue Jules-Hetzel, 92190 Meudon, France

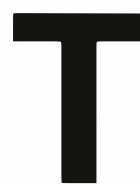
fort accroissement de ces réserves crée une situation pathologique appelée obésité qui correspond à une augmentation de la taille et du nombre des adipocytes. L'obésité est une maladie métabolique dont l'aspect visible complique le traitement. Le premier aspect de l'obésité est lié à la définition personnelle du poids souhaité et à l' "image de soi " : être obèse, c'est devoir affronter une image de soi non superposable à celle que renvoie les médias, le cinéma etc., la minceur y étant présentée comme un idéal. Ainsi, tout en souffrant organiquement, beaucoup d'obèses souffrent psychologiquement de leur état. Le second aspect de l'obésité est sa définition médicale: l'obésité est une maladie grave. Elle accroît très significativement le risque de mortalité et est associée à une forte prévalence des hyperlipémies, des maladies cardiovasculaires (insuffisance coronarienne, hypertension, thromboses veineuses), du diabète, des affections pulmonaires, des affections rénales, biliaires et ostéo-articulaires et de certains cancers (cancer de l'endomètre et cancer du sein). Dans les pays industrialisés, plus de $20 \%$ de la population est obèse et ce chiffre s'accroît chaque année. C'est donc un phénomène d'une grande fréquence et un véritable problème de santé publique qui doit mobiliser médecins, chercheurs et nutritionnistes. La masse graisseuse représente 25 à $30 \%$ du poids cor- porel chez la femme et 15 à $25 \%$ chez l'homme. Pour apprécier un surpoids, on utilise généralement l'indice de masse corporelle (poids en $\mathrm{kg} /$ carré de la hauteur en $\mathrm{m}^{2}$ ). A partir de $27 \mathrm{~kg} / \mathrm{m}^{2}$ il y a surpoids, audelà de $30 \mathrm{~kg} / \mathrm{m}^{2}$ il s'agit d'une obésité certaine. En fait, l'obésité est un syndrome hétérogène et multifactoriel. Les cliniciens savent maintenant qu'il existe plusieurs types d'obésité et qu'il convient de parler "des obésités ".

Par comparaison à d'autres domaines de la recherche biomédicale, la recherche sur l'obésité est en retard parce que cette maladie difficile à traiter, n'a pas toujours été considérée avec beaucoup d'interêt, la réponse donnée aux malades étant souvent la même : "mangez-moins !". La situation a évolué et parallèlement au développement de services médicaux spécialisés, l'identification des mécanismes conduisant à l'obésité fait actuellement l'objet de recherches dans de nombreux pays. La régulation du poids corporel est due à un équilibre entre les entrées d'énergie (calories alimentaires) et les utilisations d'énergie (métabolisme de base, exercice, thermogenèse). Toute augmentation de la prise alimentaire ou toute diminution de la dépense énergétique se traduit par un accroissement de la masse graisseuse. Il ne fait aucun doute que la suralimentation (trop d'aliments, trop de repas, trop de graisses) est un facteur essentiel dans l'établissement de l'obésité. Cependant, le débat est de savoir si cette suralimentation est la cause première de l'obésité ou si elle est secondaire à un autre 
trouble. Par ailleurs, diverses études ont montré qu'un faible défaut de thermogenèse post-prandiale chez certains obèses pouvait contribuer à l'élévation des réserves lipidiques. Comprendre et traiter les obésités, c'est faire des progrès dans la connaissance des mécanismes de contrôle de la prise alimentaire, des mécanismes de détermination et de différenciation des préadipocytes, du déterminisme de la résistance à l'insuline ; c'est aussi faire une analyse pharmacologique des adipocytes et essayer d'identifier les gènes causant ou facilitant les obésités. Au cours des vingt dernières années, une grande partie de la recherche sur l'obésité a été faite en utilisant des modèles animaux, tels les rongeurs génétiquement obèses, ou rendus obèses par manipulation nutritionnelle ou lésion de l'hypothalamus ventro-médian [1]. Des progrès importants dans la compréhension des étapes de différenciation adipocytaire ont aussi été faits [2]. Récemment, la biologie moléculaire à permis un certain nombre d'avancées qui sont commentées ici.

\section{Obésité et génétique}

La comparaison d'individus prenant facilement du poids et d'individus ayant un gros appétit et restant minces a, depuis toujours, suscité des discussions entre les premiers qui trouvent cela injuste et les seconds qui semblent facilement brûler les excès alimentaires. Claude Bouchard et ses collègues à Québec ont démontré qu'en réponse à une consommation alimentaire donnée, certains individus ont beaucoup plus de risque de prendre du poids que d'autres. Ils ont calculé que l'héritabilité génétique intervient pour 5 à $40 \%$ dans le développement de la masse graisseuse [3]. Cependant, la situation est complexe car au moins vingt gènes répartis sur douze chromosomes participent au contrôle génétique de la teneur en graisse. Parmi ces gènes se trouvent ceux de la sous-unité $\alpha$ de l'ATP-ase $\mathrm{Na}^{+} / \mathrm{K}^{+}$, du récepteur adrénergique $\alpha 2$, de l'adipsine et de la protéine décou- pling protein). A côté de cette situation complexe dans l'espèce humaine, d'autres équipes essaient d'identifier le gène unique responsable de l'obésité des souris $o b / o b, d b / d b$ et de celle des rats $f a / f a$. L'identification d'un tel gène, dont des résultats préliminaires suggèrent qu'il est transcrit dans l'hypothalamus, est attendue avec impatience.

\section{Les mécanismes thermogéniques et les fuites de protons}

Des travaux effectués chez les rongeurs ont démontré que les adipocytes bruns peuvent oxyder une partie des substrats alimentaires et dissiper cette énergie sous forme de chaleur [4-6]. Récemment, le ciblage du gène codant pour la toxine diphtérique dans les adipocytes bruns de souris transgéniques a abouti à la destruction du tissu adipeux brun chez ces animaux et à l'installation d'une obésité sévère, accompagnée de résistance à l'insuline $\left(\mathrm{m} / \mathrm{s} n^{\circ} 2\right.$, vol. 10 , p. 232, [7]). Ce travail a confirmé l'importance de l'activité thermogénique du tissu adipeux brun dans la régulation de la masse adipeuse des rongeurs (le rôle du tissu adipeux brun chez l'homme adulte n'est pas démontré). L'activité thermogénique des adipocytes bruns est principalement due à une protéine spécifique, l'UCP [5]. Un polymorphisme du gène $U C P$ humain a été découvert ; ce polymorphisme est curieusement associé à une différence importante de capacité d'accroître le pourcentage corporel de graisses au cours du temps [8]. Il est donc possible que la thermogenèse du tissu adipeux brun et le gène $U C P$ soient impliqués dans l'équilibre énergétique humain. Par ailleurs, les bioénergéticiens ont parfaitement démontré que le couplage entre réactions exergoniques et réactions endergoniques est dû à l'établissement d'un gradient de protons de part et d'autre de la membrane interne mitochondriale. Toute fuite de protons diminue le rendement de conservation d'énergie sous forme d'ATP [9]. C'est d'ailleurs ce qui explique l'activité découplante de
l'UCP. Il n'est donc pas absurde de tenter d'expliquer des différences d'utilisation et de stockage de l'énergie entre les individus par des niveaux variés de perméabilité aux protons de leurs mitochondries.

\section{Le récepteur} adrénergique $\beta 3$

Un autre point qui agite les passions parmi les personnes intéressées par la dépense énergétique et l'obésité est la découverte récente du récepteur adrénergique $\beta 3$ et le clonage du gène correspondant chez l'homme et les rongeurs [10]. Cette découverte est une retombée de la recherche sur les adipocytes bruns de rongeurs. En fait, il semble que ce récepteur soit uniquement ou très principalement exprimé dans les adipocytes bruns et blancs de ces animaux. Il y a donc une formidable possibilité d'atteindre pharmacologiquement ces adipocytes. De nombreuses compagnies pharmaceutiques sont en train de synthétiser et de tester des agonistes ß3. Ces molécules activent la thermogenèse dans les adipocytes bruns et la lipolyse dans les adipocytes blancs des rongeurs et constituent donc des médicaments potentiels pour le traitement de l'obésité. Cependant, aucun agoniste $\beta 3$ actif chez les rongeurs ou les chiens n'est, semble-t-il, actif chez l'homme ; par ailleurs un débat très vif s'est installé entre les équipes qui croient à l'existence du récepteur adrénergique $\beta 3$ chez l'homme et celles qui pensent qu'un tel récepteur n'est présent en quantité que chez les rongeurs. La mise au point de nouveaux agonistes interagissant avec la forme humaine du récepteur devrait permettre de conclure.

\section{Tissus adipeux, sécrétion et cytokines}

On a récemment découvert que les tissus adipeux produisent et sécrètent de nombreux facteurs, tels des œestrogènes, certaines prostaglandines, la monobutyrine, l'IGF1, l'apolipoprotéine $\mathrm{E}$, les facteurs $\mathrm{D}$ (ou adipsine), C3 et B du complément, l'angiotensinogène, le TNF- $\alpha$. Les adipocytes pourraient donc avoir d'autres fonc- 
tions que le seul métabolisme lipidique. Ces résultats récents suggèrent l'existence de liens entre tissus adipeux, hypertension, système immunitaire. La production adipocytaire de TNF- $\alpha$ est accrue chez les rongeurs et humains obèses [11]. De plus, cette cytokine diminue l'activité kinasique du récepteur musculaire de l'insuline. Il s'agit, peut-être, d'un début d'explication d'un type de mécanisme de résistance à l'insuline. Ces résultats peuvent constituer une nouvelle voie d'approche du traitement de la résistance à l'insuline si fréquemment associée à l'obésité.

\section{Les effets transcriptionnels des acides gras}

I.es graisses alimentaires facilitent le développement des tissus adipeux. Cela est-il la conséquence d'un simple stockage des acides gras dans les adipocytes? P. Grimaldi, E. Zoubir et G. Ailhaud ont démontré que les acides gras sont des activateurs transcriptionnels de plusieurs gènes impliqués dans le métabolisme lipidique au sein des adipocytes [12]. Récemment, la même équipe a cloné une protéine/facteur transcriptionnel qui pourrait relayer l'action des acides gras, il s'agit d'un membre de la famille des PPAR (peroxisome proliferator-activated receptor) ([13] et $\mathrm{m} / \mathrm{s}$ $n^{\circ}$ 3, vol. 8, p. 294). Cet effet transcriptionnel des acides gras signifie que les lipides de l'alimentation peuvent directement agir sur des gènes impliqués dans la différenciation et le métabolisme adipocytaire.

En conclusion, la recherche dans le domaine de l'obésité est en effervescence. Sans oublier le rôle essentiel des chercheurs étudiant les mécanismes intimes du contrôle de la prise alimentaire (en particulier l'importance de la galanine et du peptide Y pour le contrôle de l'appétit [14]), la poursuite des recherches citées et l'ouverture de nouveaux champs de recherche (telles les fonctions des cellules endothéliales des dépôts adipeux), conduiront à des progrès significatifs dans la connaissance de la biologie des tissus adipeux et des obésités

\section{RÉFÉRENCES}

1. Jeanrenaud B. Dysfonctionnement du système nerveux, obésité et résistance à l'insuline. médecine/sciences $1987 ; 3$ : 403-10.

2. Cornelius P, MacDougald OA, Lane MD. Regulation of adipocyte development. Annu Rev Nutr 1994 ; 14 : 99-129.

3. Bouchard C, Pérusse L. Heredity and body fat. Annu Rev Nutr 1988 ; 8 : 259-77.

4. Rothwell Nj, Stock MJ. A role for brown adipose tissue in diet-induced thermogenesis. Nature $1979 ; 218: 31-5$.

5. Ricquier D. Thermogenèse et obésité : mécanismes moléculaires. médecine/sciences $1985 ; 1: 147-53$.

6. Seydoux J. Défaut de thermogenèse et obésité. médecines/sciences 1987; 3 : 387-93.

7. Lowell BB, Susulic VS, Hamann A, Lawitts JA, Himms-Hagen J, Boyer BB, Kozak LP, Flier JS. Development of obesity in transgenic mice after genetic ablation of brown adipose tissue. Nature 1993 ; 366 : $740-2$.

8. Oppert JM, Vohl MC, Chagnon M, Dionne FT, Cassard-Doulcier AM, Ricquier D, Pérusse L, Bouchard C. DNA polymorphism in the uncoupling protein (UCP) gene and human body fat. Int J Obes 1994 ; $18: 526-31$.

9. Brand MD, Chien LF, Ainscow EK, Rolfe DFS, Porter RK. The causes and functions of mitochondrial proton leak. Biochim Biophys Acta 1994 ; 1187 : 132-9.

10. Emorine L, Strosberg AD. Structure et fonction du récepteur adrénergique $\beta 3$. médecine/sciences $1993 ; 9: 1228-35$.

11. Hotamisligil GS, Shargill NS, Spiegelman BM. Adipose expression of tumor necrosis factor- $\alpha$ : direct role in obesity-linked insulin resistance. Science $1993 ; 259: 87-90$.

12. Amri E, Bertrand B, Ailhaud G, Grimaldi $P$. Regulation of adipose cell differentiation I. Fatty acids are inducers of the aP2 gene expression. J Lipid Res 1991 ; 32 : 144956.

13. Latruffe N. Les peroxysomes et la prolifération cellulaire ou la prise en considération d'un organite méconnu. médecine/sciences $1992 ; 8: 239-48$.

14. Castan I, Valet $P$, Lafontan $M$. Le récepteur du PYY : un élément important du svstème antilipolytique de l'adipocyte. médeci$n e /$ sciences $1994 ; 10$ : 196-201. 